\title{
Statin use and all-cause mortality in people living with HIV: a systematic review and meta-analysis
}

\author{
Olalekan A. Uthman ${ }^{1 *}$, Chidozie Nduka², Samuel I. Watson ${ }^{1}$, Edward J. Mills ${ }^{3}$, Andre P. Kengne ${ }^{4,5}$, Shabbar S. Jaffar ${ }^{6}$,
} Aileen Clarke ${ }^{2}$, Tahereh Moradi ${ }^{7}$, Anna-Mia Ekström ${ }^{8,9}$ and Richard Lilford ${ }^{1}$

\begin{abstract}
Background: It is unknown whether statin use among people living with HIV results in a reduction in all-cause mortality. We aimed to evaluate the effect of statin use on all-cause mortality among people living with HIV.
\end{abstract}

Methods: We conducted comprehensive literature searches of Medline, Embase, CINAHL, the Cochrane Library, and cross-references up to April 2018. We included randomised, quasi-randomised trials and prospective cohort studies that examined the association between statin use and cardio-protective and mortality outcomes among people living with HIV. Two reviewers independently abstracted the data. Hazard ratios (HRs) were pooled using empirical Bayesian random-effect meta-analysis. A number of sensitivity analyses were conducted.

Results: We included seven studies with a total of 35,708 participants. The percentage of participants on statins across the studies ranged from 8 to $35 \%$. Where reported, the percentage of participants with hypertension ranged from 14 to $35 \%$ and 7 to $10 \%$ had been diagnosed with diabetes mellitus. Statin use was associated with a 33\% reduction in all-cause mortality (pooled $H R=0.67,95 \%$ Credible Interval 0.39 to 0.96 ). The probability that statin use conferred a moderate mortality benefit (i.e. decreased risk of mortality of at least $25 \%$, HR $\leq 0.75$ ) was $71.5 \%$. Downweighting and excluding the lower quality studies resulted in a more conservative estimate of the pooled HR.

Conclusion: Statin use appears to confer moderate mortality benefits in people living with HIV.

Keywords: Statin, HIV, Mortality

\section{Background}

Although life expectancy for people living with HIV has improved dramatically over the past two decades following the introduction of highly active antiretroviral therapy, mortality rates remain higher than in the general population $[1,2]$. As life expectancy has increased, the incidence of non-communicable diseases (NCDs) including cardio-metabolic disorders has also disproportionately increased along with risky behaviours such as smoking, and has been identified as a major cause of excess mortality [3]. Dyslipidaemias are perhaps the most common and most studied cardio-metabolic disorders affecting people with HIV [4]. There are strong reasons

\footnotetext{
* Correspondence: olalekan.uthman@warwick.ac.uk

${ }^{1}$ Warwick-Centre for Applied Health Research and Delivery (WCAHRD), Warwick Medical School, University of Warwick, Coventry CV4 7AL, UK Full list of author information is available at the end of the article
}

to hypothesise that statins are effective in reducing cardiovascular events in people living with HIV. First, they are effective in many groups and among high-risk people who do not have HIV [5-17]. Second, they have been shown to reduce dyslipidaemia in HIV-infected people [18]. Third, they have been shown to act anti-inflammatory agent $[19,20]$ and improve surrogate markers for cardiovascular events, such as carotid intima-media thickness [21] and coronary artery plaque volume in people living with HIV [22].

Although studies of statins in HIV have evaluated subclinical CVD, none has evaluated associations between statin use and hard CVD endpoints. This includes nonfatal CVD events such as MI and stroke as well as CVD mortality. Thus, the logical next step would be to evaluate statin use and hard CVD endpoints for HIV+ persons. To date, no randomised trials have been

(C) The Author(s). 2018 Open Access This article is distributed under the terms of the Creative Commons Attribution 4.0 International License (http://creativecommons.org/licenses/by/4.0/), which permits unrestricted use, distribution, and 
published on this topic. However, analyses of hard CVD endpoints, including CVD mortality, may be underpowered due to insufficient data on hard CVD endpoints and/or cause-specific mortality in $\mathrm{HIV}+$ cohorts and trial registries where statin use was assessed. As a result, this study sought to leverage the best currently available data and primarily evaluate overall mortality among HIV + persons taking vs. not taking statins, with the evaluation of CVD mortality as an exploratory analysis. Evidence regarding the potential benefits of a particular intervention is often available from a variety of disparate sources. When considering the benefit of an intervention - particularly in the absence of any RCTs addressing the relevant question - that 'real-world' evidence from non-randomized studies should also be considered [23]. We aimed to examine whether statin use is associated with all-cause mortality using a systematic review and meta-analysis of prospective cohort studies.

\section{Methods \\ Eligibility criteria}

To be included, studies had to meet the following selection criteria:

Types of studies: Randomised, quasi-randomised trials and prospective cohort studies.

Types of participants: adult ( $>18$ years) people living with HIV (PLHIV) of either sex.

Types of intervention: Any form of statin use regardless of indication, including but not limited to primary or secondary prevention of cardiovascular disease.

Types of comparator: no statin or placebo

Types of outcome measures: All-cause mortality.

\section{Information sources and search strategy}

We conducted a thorough literature search to identify relevant studies. We searched electronic databases of Medline, CINAHL and Web of Science from 1980 to April 2018 without applying any language restriction. We searched for abstracts of relevant conference proceedings from the National Library of Medicine Gateway. In addition, the bibliographies of retrieved articles were examined for pertinent studies. The full Medline search strategy is shown in Additional file 1: Appendix S1.

\section{Study selection and data extraction}

Two authors (OU and $\mathrm{NC}$ ) evaluated the eligibility of studies obtained from the literature search using a predefined protocol and worked independently to scan all abstracts and obtain the full texts of each selected article. For each included study, details on design, population characteristics, intervention and outcome measures were extracted, and risk of bias was evaluated. Any discrepancies between the authors were resolved through discussion and involving a third author. Two authors (OU and $\mathrm{NC}$ ) independently extracted data.

\section{Risk of bias assessment}

We used the Cochrane tool Risk Of Bias In Non-randomised Studies - of Interventions (ROBINS-I) to assess the risk of bias of included studies (see Additional file 1: Appendix S2) [24]. We assessed risks of bias in the following seven domains, facilitated by consideration of pertinent "signalling" questions, bias due to: confounding, selection of participants, measurement of interventions, departures from intended interventions, missing data, measurement of outcomes, selection of reported results. Within each domain, we rated risk of bias as "low" (comparable to a well performed randomised trial), "moderate" (sound for an observational study), "high" (there are important problems), or "very high" (the study is too problematic to provide useful evidence). The judgements within each domain were carried forward to an overall risk of bias judgement across bias domains.

\section{Statistical analysis}

For the main analysis, we performed Bayesian randomeffects meta-analysis [25] with a prior based on expected heterogeneity to pool the hazard ratio (HR) estimates for the association between statin use and all-cause mortality among studies [26]. We selected random-effects meta-analysis on account of anticipated heterogeneity in study population and methodology [26]. Treatment effect measures, the hazard ratio and odds ratio were log transformed to reduce skewness. We combined hazard ratio (HR) and odds ratio (OR) in the meta-analysis. HR and OR can be interpreted similarly if the underlying assumption of a generally low event risk $(<20 \%)$ is true [27].

We performed a meta-regression analysis to explore the relationship between the following study-level factors and reported treatment effects: sample size, publication year, cohort follow-up period, percentage of statin use, percentage male, mean age and study location (Europe vs. North America). Given the low number of studies identified in the study univariate meta-regressions were estimated as opposed to multivariate. All models were estimated using STAN and R [28].

\section{Sensitivity analyses}

A number of sensitivity analyses were conducted to examine the robustness of the results to study quality and modelling assumptions. First, we assigned 'quality weights' to the studies on the basis of the risk of bias assessment [29]. The quality weights can be interpreted as the proportion of variance of a studies results not attributable to bias. Two sets of weights were used: (i) studies at high risk of bias were assigned a weight of $20 \%$ and studies at a moderate/low risk were assigned a weight of $70 \%$; (ii) the 
respective weights for high risk and low/moderate risk were 50 and $80 \%$. Second, we re-estimated the model excluding studies deemed as being at high risk of bias (a quality weight of zero). Third, we examined the sensitivity of the results to the choice of prior distribution. We used an informative 'sceptical' prior distribution based on the principle that 'Most clinically important interventions are likely to reduce the relative risk of all-cause mortality by about 10-20\%.' The sceptical prior specifies that there was only a $5 \%$ probability that the HR was less than 0.75 [30].

This systematic review was reported according to the Preferred Reporting Items for Systematic Reviews and Meta-analyses (PRISMA) guidelines [31] (Additional file 1: Appendix S3).

\section{Results}

Study selection and characteristics

The process of study selection is shown in Fig. 1. Overall, the literature searches of databases yielded 615 articles. After review of abstracts and titles, 11 articles were selected for critical reading. Four studies did not meet the inclusion criteria [32-35] as no relevant outcomes were reported. Seven studies with a total of 35,708 participants were included [36-42]. The characteristics of the included studies are shown in Table 1. The studies were conducted between 1995 and 2015 and published between 2011 and 2015. Five were reported as full-text journal articles [38-42], and two were presented as conference abstracts [36, 37]. All the studies were from high-income countries; four from the USA. The other studies were conducted in Denmark, France and Spain. The median number of participants was 1738 and ranged from 438 to 25,884 . All the seven studies were cohort studies [36-42]. The mean age at entry of the participants ranged from 39 to 51 years and the proportions of males included in the studies ranged from $67 \%$ to as much as $98 \%$ (from US Veterans Affairs' Clincial Case Registry). The percentage of participants on statins

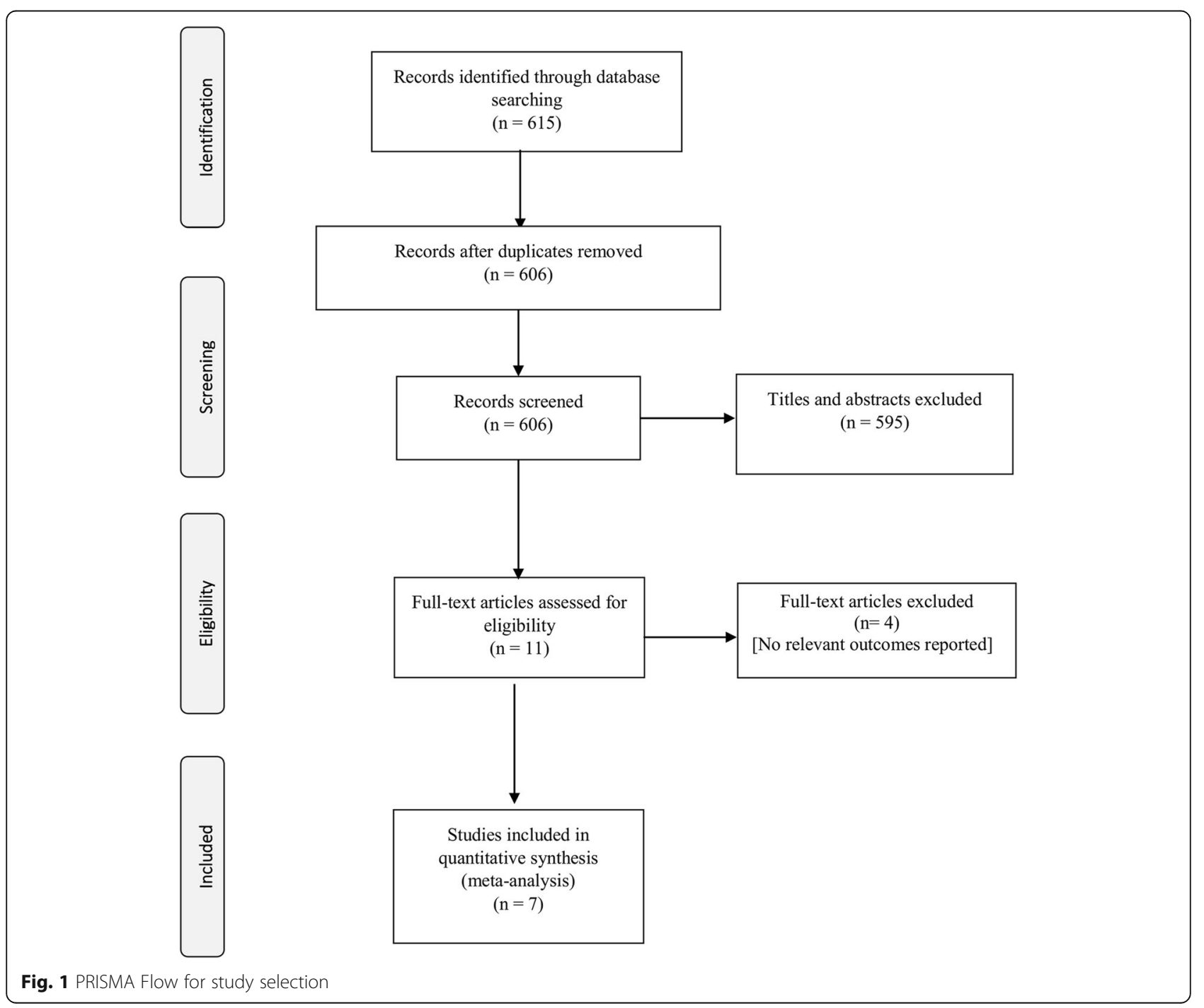




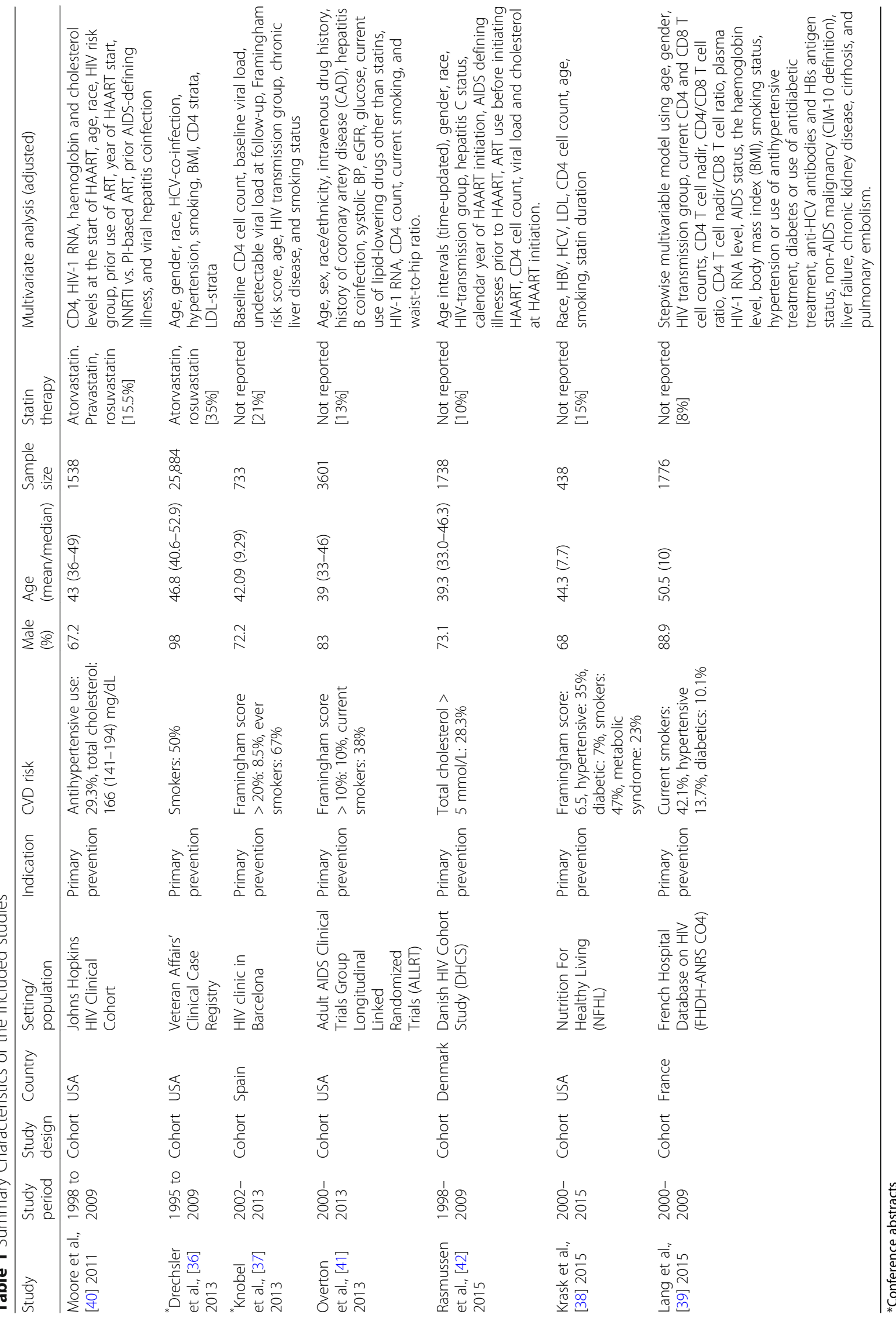


across the studies ranged from 8 to $35 \%$. None of the study reported statin type. When reported, the percentage of participants with hypertension ranged from 14 to $35 \%$ and 7 to $10 \%$ had been diagnosed with diabetes mellitus. None of the studies reported rates of adherence to statins use.

\section{Quality assessment of included studies}

A summary of the risk of bias assessment in the included studies is shown in Additional file 1: Table S1. The risk of bias due to confounding was moderate all the seven studies. The bias in selection of participants was moderate in four studies [36, 38, 41, 42] and serious in three studies [37, 39, 40] For Knobel and colleagues study [37], the Framingham cardiovascular risk score above $20 \%$ was significantly higher among those on statin compared with those not on statins $(21.4 \%$ versus $5.0 \%, \mathrm{RR}=2.95,95 \% \mathrm{CI} 2.22$ to 3.92 ) and those on statin had significantly higher median total cholesterol (231 versus $178 \mathrm{mg} / \mathrm{dL}$ ). Similarly, for Lang et al. [39], the proportion of participants with hypertension $(29.7 \%$ versus $12.4 \%)$ and type 2 diabetes mellitus (21.7\% versus 9.1\%) was significantly higher among those exposed to statins compared with those not on statins at baseline. For Moore and colleagues study [40], the proportion of participants on antihypertensive medications was significantly higher among those on statin compared with those not on statins (46.3\% versus $25.6 \%$ ). The bias in measurement of interventions due to departures from intended interventions and due to missing data were moderate all the seven studies. The bias in measurement of outcomes was low in all studies, because the outcome of study was death ascertained via adequate record linkages [36-42]. The bias in selection of reported studies was rated serious in the two studies published as conference abstracts [36, 37] and moderate in the remaining five studies. Overall risk of bias was moderate in three studies and serious in four studies.

\section{Effects of statins on all-cause mortality}

The Bayesian random-effects meta-analysis yielded a pooled HR of 0.67 (95\% CrI 0.39 to 0.96 ) in the risk of all-cause mortality; a $33 \%$ reduction in absolute risk (Fig. 2). However, 95\% prediction interval for the pooled HR contains values greater than 1 ( 0.21 to 1.76$)$, which suggests that although on average statins seem to be effective in reducing all-cause mortality, not all future individual studies can be expected to show all-cause mortality benefits of statins.

Table 2 and Additional file 1: Table S2 reports the estimated pooled HRs from the sensitivity analyses. Down-weighting the lower quality studies resulted in a more conservative estimate of the pooled HR. The pooled HRs from the two weighting schemes were 0.82 $(0.49,1.35)$ and $0.76(0.50,1.13)$. Excluding low quality also resulted in a more conservative estimate of the pooled HR. The 'sceptical' prior resulted in a posterior mean estimate of the pooled HR of $0.88(0.69,1.14)$.

In a series of meta-regression analyses, none of the study level factors were associated with treatment effect estimates (Table 3).

Only two studies [37, 40] reported cardiovascular disease mortality as an outcome. The pooled HR from these two studies was $1.23(0.52,2.61)$. Only two studies $[36,41]$ reported cardiovascular disease event rates as on outcome. The pooled HR was $0.69(0.37,1.62)$.

\section{Discussion}

\section{Main findings}

To our knowledge, this systematic review and meta-analysis, comprising seven observational studies with more than 35,000 HIV-infected participants, is the first to examine the effect of statin therapy on all-cause mortality in people living with HIV. Overall, the findings support the expectation that statins confer mortality benefit. However, due to the limited evidence currently available, we can draw no conclusions as to effectiveness of statins on cardiovascular disease mortality and

$\begin{array}{lr}\text { Study } & \text { Sample size } \\ \text { Drechsler et al, 2013 } & 25884 \\ \text { Knobel et al, 2013 } & 733 \\ \text { Krsak et al, 2015 } & 438 \\ \text { Lang et al,37 2015 } & 1776 \\ \text { Moore et al, 2001 } & 1538 \\ \text { Overton et al, 2015 } & 3601 \\ \text { Rasmussen et al, 2015 } & 1738 \\ & \\ \text { Bayes overall (non-informative prior) } \\ \text { Predictive Interval }\end{array}$

Predictive Interval

\section{$0.67[0.39,0.96]$ \\ $[0.21,1.76]$}

Fig. 2 Forest plot of association between statins use and all-cause mortality 
Table 2 Estimated pooled HRs from the sensitivity analyses

\begin{tabular}{lllll}
\hline & Posterior mean $(95 \% \mathrm{Crl})$ & Number of studies & $\mathrm{I}^{2}$ & Probability HR $<1$ \\
\hline Main analysis & $0.67(0.39,0.96)$ & 7 & $63 \%$ & $97 \%$ \\
Down-weight low quality studies $(20 \% / 70 \%)^{\mathrm{a}}$ & $0.82(0.49,1.35)$ & 7 & $49 \%$ & $80 \%$ \\
Down-weight low quality studies $(50 \% / 80 \%)^{\mathrm{b}}$ & $0.76(0.50,1.13)$ & 7 & $43 \%$ & $92 \%$ \\
Exclude low quality studies & $0.81(0.49,1.37)$ & 4 & $45 \%$ & $81 \%$ \\
'Sceptical' prior & $0.88(0.69,1.14)$ & 7 & $62 \%$ & $85 \%$ \\
\hline
\end{tabular}

cardiovascular events. Nevertheless, the results of our meta-analysis usefully extend previously published meta-analyses of statin use in other high-risk groups such as the recent pivotal collaborative meta-analysis of individual participants' data by the Cholesterol Treatment Trialists' (CTT) [7] based on 174, 000 individuals, that reported reductions of approximately $10 \%$ in all-cause mortality for both women (risk ratio, 0.91; 99\% CI 0.84-0.99) and men (risk ratio, 0.90, 99\% 0.86-0.95). However, there has been conflicting literature about the association of statin use with all-cause mortality in other population [11, 13]. Ray and colleagues conducted a meta-analysis of 11 RCTs involving 65,2229 participants to examine the effect of statin use on all-cause mortality among intermediate to high-risk individuals without a history of CVD [11]. They found that the use of statins in this high-risk primary prevention setting was not associated with a statistically significant reduction (risk ratio, $0.91 ; 95 \%$ confidence interval, $0.83-1.01$ ) in the risk of all-cause mortality [11]. Taylor and colleagues conducted Cochrane review to assess the effects, both harms and benefits, of statins in in adults with no restrictions on total, low density lipoprotein (LDL) or high-density lipoprotein (HDL) cholesterol levels, and where $10 \%$ or less had a history of CVD [13]. They included 18 RCTs involving 56,934 participants and found that all-cause mortality was reduced by statins (odds ratio $0.86,95 \%$ confidence interval, 0.79 to 0.94 ) [13].

PLHIV are now living longer than because of effective treatment with antiretroviral therapy [43]. However, the increased life expectancy is now associated with increased prevalence of chronic conditions such as

Table 3 Study level factors associated with treatment effect estimates, meta-regression analyses

\begin{tabular}{ll}
\hline Factor & $\begin{array}{l}\text { Ratio of Hazard Ratio } \\
\text { (95\% Credible Interval) }\end{array}$ \\
\hline Sample size (per 1000 people) & $1.02(0.97$ to 1.06$)$ \\
Publication year & $1.20(0.85$ to 1.71$)$ \\
Cohort follow-up period & $1.16(0.93$ to 1.38$)$ \\
Statin use (\%) & $1.01(0.95$ to 1.06$)$ \\
Male (\%) & $1.02(0.98$ to 1.05$)$ \\
Age (mean, years) & $1.08(0.98$ to 1.18$)$ \\
Europe vs. North America studies & $0.94(0.31$ to 2.27$)$ \\
\hline
\end{tabular}

cardiovascular disease [43]. It has now been documented in the literature that PLHIV are at increased risk of developing CVD than non-HIV patients [43-45]. In addition, there is disparities in the quality of CVD care between PLHIV and uninfected adults [46]. Ladapo and colleagues found that "Physicians generally underused guideline-recommended cardiovascular care and were less likely to prescribe aspirin and statins to HIV-infected patients at increased risk-findings that may partially explain higher rates of adverse cardiovascular events among patients with HIV". [46] Implementation of intensive lifestyle modification in PLHIV may help reduce CVD mortality and morbidity [43]. The frequency and consistency of clinical encounters with PLHIV may provide a unique opportunity to provide them with continuous assistance to help behaviour changes to prevent CVD risk [43]. Given the crucial need for prevention of cardiovascular disease in PLHIV, there is a need for a multi-morbidity trials to definitively assess the efficacy of statins as a primary prevention strategy for cardiovascular disease in this at-risk population [47, 48], especially in resource-limited settings that bear the highest burden of HIV. Furthermore, low income settings are now experiencing an epidemiological transition from infectious diseases to chronic diseases, [49] as a result of dramatic changes in diet and lifestyle. The epidemiological transition in LMICs is happening over a shorter time frame than that experienced historically by high-income countries [50].

\section{Study limitations and strengths}

Strengths of this study include the comprehensive searches of databases to ensure that all relevant, published studies were identified. We used a Bayesian approach that allows us to utilize informative prior information. The main limitation of our study is that the nature of the literature is entirely observational. Statistical adjustment cannot exclude existing confounding, such as confounding by indication or variations in other patient or clinician level variability that might be independently associated with the outcome. Counter-intuitively, there is some evidence that statin may increase risk of CVD mortality, but perhaps not surprising because it likely reflects some confounding by therapy indication - people prescribed statins may be more likely to have pre-existing CVD or be at greater risk for CVD, and this risk is unlikely to be fully 
accounted for through multivariable adjustment. Thus, factors leading to these people being prescribed statins may be responsible for their apparently elevated CVDrelated mortality rather than their actual use of statins. Statins are a large constellation with different results depending upon the type and dose of statin therapy. As these data were not reported, it was not possible to conduct sensitivity analyses to stratify by different types and doses of statins. In addition, the pooled association should be interpreted with caution because it is derived from observational studies. Our study found high $I^{2}$ values as measures of heterogeneity. However, it is worth noting that the $I^{2}$ measurement offers inflated estimates when dealing with non-comparative studies. Meta-regression analyses are prone to ecological fallacy (aggregate bias) and may have low power to detect an association [51-53]. We did not conduct tests for publication bias because we included observational studies and we are aware that many patients outside these studies receive statin treatment, so there is, by definition, publication bias. Finally, the fact that most included studies were observational may explain a larger treatment effect observed in our analysis than that observed among RCTs in non-HIV patients [7].

\section{Conclusions}

In summary, based on pooled data on 35,708 HIV-infected participants from seven observational studies, we observed that statin therapy may have an important mortality benefit in people living with HIV, accounting for an estimated 33\% reduction in all-cause mortality. While awaiting a definitive answer from on-going trials and long-term observational studies about the benefits of statin as a primary prevention for cardiovascular disease and all-cause mortality, our findings are timely and reassuring. They reinforce the notion that lowering lipid levels is likely to be associated with a reduction in all-cause mortality in people living with HIV as it is in other high-risk groups.

\section{Additional file}

Additional file 1: Appendix S1. Medline Search Strategy. Appendix S2. Risk bias assessment. Appendix S3. PRISMA checklist. Table S1. Riskof-bias assessment of included studies. Table S2. Estimated pooled HRs from the sensitivity analyses. (DOCX $41 \mathrm{~kb}$ )

\footnotetext{
Acknowledgements

Samuel Watson, Aileen Clarke and Richard Lilford are supported by the NIHR CLAHRC West Midlands initiative. Olalekan Uthman, Samuel Watson, and Richard Lilford are supported by the National Institute for Health Research using Official Development Assistance (ODA) funding. The views expressed in this publication are those of the author(s) and not necessarily those of the NHS, the National Institute for Health Research or the Department of Health and Social Care.
}

Availability of data and materials

All data generated or analysed during this study are included in this published article and its additional file.

\begin{abstract}
Authors' contributions
OAU led protocol design, study design, the literature review, data extraction, statistical analysis, data interpretation, article preparation, article review, and correspondence. CN, SIW, RL contributed to protocol design, study design, the literature review, data extraction, statistical analysis, data interpretation, article preparation, article review, and correspondence. EJM, APK, SSJ, AC, TM, AME contributed to data interpretation, article preparation, article review, and correspondence. All authors have read and approved the final manuscript.
\end{abstract}

Ethics approval and consent to participate

Not applicable.

\section{Competing interests}

The authors declare that they have no competing interests.

\section{Publisher's Note}

Springer Nature remains neutral with regard to jurisdictional claims in published maps and institutional affiliations.

\section{Author details}

${ }^{1}$ Warwick-Centre for Applied Health Research and Delivery (WCAHRD), Warwick Medical School, University of Warwick, Coventry CV4 7AL, UK. ${ }^{2}$ Division of Health Sciences, Warwick Medical School, University of Warwick, Coventry, UK. ${ }^{3}$ McMaster University, Hamilton, Canada. ${ }^{4}$ Non-Communicable Diseases Research Unit, South African Medical Research Council, Cape Town, South Africa. ${ }^{5}$ Department of Medicine, University of Cape Town, Cape Town, South Africa. ${ }^{6}$ Liverpool School of Tropical Medicine, Dept of International Public Health, Liverpool, UK. ${ }^{7}$ Institute of Environmental Medicine, Division of Epidemiology, Karolinska Institutet Stockholm, Stockholm, Sweden.

${ }^{8}$ Department of Public Health (IHCAR), Karolinska Institutet, Stockholm, Sweden. ${ }^{9}$ Department of Infectious Diseases, Karolinska University Hospital, Stockholm, Sweden.

Received: 3 October 2017 Accepted: 23 May 2018

Published online: 05 June 2018

\section{References}

1. Global, regional, and national life expectancy, all-cause mortality, and causespecific mortality for 249 causes of death, 1980-2015: a systematic analysis for the Global Burden of Disease Study 2015. Lancet (London, England). 2016;388(10053):1459-544.

2. Wandeler G, Johnson LF, Egger M. Trends in life expectancy of HIV-positive adults on antiretroviral therapy across the globe: comparisons with general population. Curr Opin HIV AIDS. 2016;11(5):492-500.

3. Hooshyar D, Hanson DL, Wolfe M, Selik RM, Buskin SE, McNaghten AD. Trends in perimortal conditions and mortality rates among HIV-infected patients. AIDS (London, England). 2007;21(15):2093-100.

4. Nduka C, Sarki A, Uthman O, Stranges S. Impact of antiretroviral therapy on serum lipoprotein levels and dyslipidemias: a systematic review and meta-analysis. Int J Cardiol. 2015;199:307-18.

5. Baigent C, Keech A, Kearney PM, Blackwell L, Buck G, Pollicino C, Kirby A, Sourjina T, Peto R, Collins R, et al. Efficacy and safety of cholesterol-lowering treatment: prospective meta-analysis of data from 90,056 participants in 14 randomised trials of statins. Lancet (London, England). 2005;366(9493):1267-78.

6. de Vries FM, Denig P, Pouwels KB, Postma MJ, Hak E. Primary prevention of major cardiovascular and cerebrovascular events with statins in diabetic patients: a meta-analysis. Drugs. 2012;72(18):2365-73.

7. Fulcher J, O'Connell R, Voysey M, Emberson J, Blackwell L, Mihaylova B, Simes J, Collins R, Kirby A, Colhoun $\mathrm{H}$, et al. Efficacy and safety of LDLlowering therapy among men and women: meta-analysis of individual data from 174,000 participants in 27 randomised trials. Lancet (London, England). 2015:385(9976):1397-405.

8. Jia M, Huang W, Li L, Xu Z, Wu L. Statins reduce mortality after non-severe but not after severe pneumonia: a systematic review and meta-analysis. J Pharm Pharm Sci. 2015;18(3):286-302. 
9. Jung JM, Choi JY, Kim HJ, Seo WK. Statin use in spontaneous intracerebral hemorrhage: a systematic review and meta-analysis. Int J Stroke. 2015;10(Suppl A100):10-7.

10. Minder CM, Blumenthal RS, Blaha MJ. Statins for primary prevention of cardiovascular disease: the benefits outweigh the risks. Curr Opin Cardiol. 2013;28(5):554-60.

11. Ray KK, Seshasai SR, Erqou S, Sever P, Jukema JW, Ford I, Sattar N. Statins and all-cause mortality in high-risk primary prevention: a meta-analysis of 11 randomized controlled trials involving 65,229 participants. Arch Intern Med. 2010;170(12):1024-31.

12. Tapia Perez JH, Yildiz OC, Schneider T, Nimsky C. Meta-analysis of statin use for the acute therapy of spontaneous intracerebral hemorrhage. J Stroke Cerebrovasc Dis. 2015;24(11):2521-6.

13. Taylor F, Huffman MD, Macedo AF, Moore TH, Burke M, Davey Smith G, Ward K, Ebrahim S. Statins for the primary prevention of cardiovascular disease. Cochrane Database Syst Rev. 2013;1:Cd004816.

14. Taylor F, Huffman MD, Macedo AF, Moore THM, Burke M, Davey Smith G, Ward K, Ebrahim S. Statins for the primary prevention of cardiovascular disease. Cochrane Database Syst Rev. 2013;(1):CD004816. https://doi.org/10. 1002/14651858.CD004816.pub5

15. Teng M, Lin L, Zhao YJ, Khoo AL, Davis BR, Yong QW, Yeo TC, Lim BP. Statins for primary prevention of cardiovascular disease in elderly patients: systematic review and meta-analysis. Drugs Aging. 2015;32(8):649-61.

16. Vrecer M, Turk S, Drinovec J, Mrhar A. Use of statins in primary and secondary prevention of coronary heart disease and ischemic stroke. Metaanalysis of randomized trials. Int J Clin Pharmacol Ther. 2003;41(12):567-77.

17. Yang M, Xie XS, Yuan WJ. A meta-analysis of the effects of statin treatment on cardiovascular events and all-cause mortality in diabetic dialysis patients. Int J Clin Exp Med. 2015;8(6):8415-24.

18. Feinstein MJ, Achenbach CJ, Stone NJ, Lloyd-Jones DM. A systematic review of the usefulness of statin therapy in HIV-infected patients. Am J Cardiol. 2015;115(12):1760-6.

19. Feinstein MJ, Bahiru E, Achenbach C, Longenecker CT, Hsue P, So-Armah K, Freiberg MS, Lloyd-Jones DM. Patterns of cardiovascular mortality for HIV-infected adults in the United States: 1999 to 2013. Am J Cardiol. 2016;117(2):214-20

20. Schoenfeld SR, Lu L, Rai SK, Seeger JD, Zhang Y, Choi HK. Statin use and mortality in rheumatoid arthritis: a general population-based cohort study. Ann Rheum Dis. 2016;75(7):1315-20.

21. Calza L, Manfredi R, Colangeli V, Trapani FF, Salvadori C, Magistrelli E, Danese I, Verucchi G, Serra C, Viale P. Two-year treatment with rosuvastatin reduces carotid intima-media thickness in HIV type 1-infected patients receiving highly active antiretroviral therapy with asymptomatic atherosclerosis and moderate cardiovascular risk. AIDS Res Hum Retrovir. 2013;29(3):547-56.

22. Lo J, Lu MT, Ihenachor EJ, Wei J, Looby SE, Fitch KV, Oh J, Zimmerman CO, Hwang J, Abbara S, et al. Effects of statin therapy on coronary artery plaque volume and high-risk plaque morphology in HIV-infected patients with subclinical atherosclerosis: a randomised, double-blind, placebo-controlled trial. Lancet HIV. 2015;2(2):e52-63.

23. Nallamothu BK, Hayward RA, Bates ER. Beyond the randomized clinical trial: the role of effectiveness studies in evaluating cardiovascular therapies. Circulation. 2008;118(12):1294-303.

24. Sterne JA, Hernan MA, Reeves BC, Savovic J, Berkman ND, Viswanathan M, Henry D, Altman DG, Ansari MT, Boutron I, et al. ROBINS-I: a tool for assessing risk of bias in non-randomised studies of interventions. BMJ (Clinical Res Ed). 2016;355:i4919.

25. Sutton AJ, Abrams KR. Bayesian methods in meta-analysis and evidence synthesis. Stat Methods Med Res. 2001;10(4):277-303.

26. Turner RM, Davey J, Clarke MJ, Thompson SG, Higgins JP. Predicting the extent of heterogeneity in meta-analysis, using empirical data from the Cochrane database of systematic reviews. Int J Epidemiol. 2012;41(3):818-27.

27. Symons MJ, Moore DT. Hazard rate ratio and prospective epidemiological studies. J Clin Epidemiol. 2002;55(9):893-9

28. Stan Development Team: Stan Modeling Language User's Guide and Reference Manual, Version 2.10.0. URL http://mc-stan.org/ [Accessed 10 Oct 2016]; 2015.

29. Thompson S, Ekelund U, Jebb S, Lindroos AK, Mander A, Sharp S, Turner R, Wilks D. A proposed method of bias adjustment for meta-analyses of published observational studies. Int J Epidemiol. 2011;40(3):765-77.

30. Higgins JP, Spiegelhalter DJ. Being sceptical about meta-analyses: a Bayesian perspective on magnesium trials in myocardial infarction. Int J Epidemiol. 2002;31(1):96-104.
31. Moher D, Liberati A, Tetzlaff J, Altman DG. Preferred reporting items for systematic reviews and meta-analyses: the PRISMA statement. Ann Intern Med. 2009;151(4):264-9. w264

32. Elsamadisi P, Cha A, Kim E, Latif S. Statin Use With the ATP III Guidelines Compared to the 2013 ACC/AHA Guidelines in HIV Primary Care Patients. J Pharm Pract. 2017;30(1):64-69.

33. Eckard AR, Meissner EG, Singh I, McComsey GA. Cardiovascular Disease, Statins, and HIV. J Infect Dis. 2016;214(Suppl 2):S83-92.

34. Ou HT, Chang KC, Li CY, Yang CY, Ko NY. Intensive statin regimens for reducing risk of cardiovascular diseases among human immunodeficiency virus-infected population: A nation-wide longitudinal cohort study 20002011. Int J Cardiol. 2017;230:592-98.

35. Lichtenstein KA, Hart RL, Wood KC, Bozzette S, Buchacz K, Brooks JT, Investigators HIVOS. Statin use is associated with incident diabetes mellitus among patients in the HIV outpatient study. J Acquir Immune Defic Syndr. 2015;69(3):306-11.

36. Drechsler H, Zhang S, Maalouf N, Cutrell J, Tebas P, Bedimo R: Impact of statin exposure on mortality and non-AIDS complications in HIV patients on HAART. Abstract 765. In: 20th conference on retroviruses and opportunistic infections march 3-6, 2013. Atlanta: Conference on Retroviruses and Opportunistic Infections (CROI); 2013.

37. Knobel H, Fratchez V, Montero M, Villar J, Lerma E, Gonzalez E, Molas E, Sorli $L$, Guerri R, Guelar A: The use of statins was associated with reduced mortality in HIV-infected patients. Abstract PE12/7. In: 14th European AIDS Conference October 16-19. Brussels; 2013.

38. Krsak M, Kent DM, Terrin N, Holcroft C, Skinner SC, Wanke C. Myocardial infarction, stroke, and mortality in CART-treated HIV patients on statins. AIDS Patient Care STDs. 2015;29(6):307-13.

39. Lang S, Lacombe JM, Mary-Krause M, Partisani M, Bidegain F, Cotte L, Aslangul E, Cheret A, Boccara F, Meynard JL, et al. Is impact of statin therapy on all-cause mortality different in HIV-infected individuals compared to general population? Results from the FHDH-ANRS CO4 cohort. PLoS One. 2015:10(7):e0133358.

40. Moore RD, Bartlett JG, Gallant JE. Association between use of HMG CoA reductase inhibitors and mortality in HIV-infected patients. PLoS One. 2011;6(7):e21843.

41. Overton ET, Kitch D, Benson CA, Hunt PW, Stein JH, Smurzynski M, Ribaudo HJ, Tebas P. Effect of statin therapy in reducing the risk of serious non-AIDSdefining events and nonaccidental death. Clin Infect Dis. 2013;56(10):1471-9.

42. Rasmussen LD, Kronborg G, Larsen CS, Pedersen C, Gerstoft J, Obel N. Statin therapy and mortality in HIV-infected individuals; a Danish nationwide population-based cohort study. PLoS One. 2013;8(3):e52828.

43. Bouvier G. Lifestyle modification and cardiovascular disease in HIV. HIV Clin. 2012;24(4):1. 5-6

44. Triant VA. Cardiovascular disease and HIV infection. Curr HIV/AIDS Rep. 2013;10(3):199-206.

45. Triant VA, Lee H, Hadigan C, Grinspoon SK. Increased acute myocardial infarction rates and cardiovascular risk factors among patients with human immunodeficiency virus disease. J Clin Endocrinol Metab. 2007;92(7):2506-12.

46. Ladapo JA, Richards AK, DeWitt CM, Harawa NT, Shoptaw S, Cunningham WE, Mafi JN. Disparities in the quality of cardiovascular care between HIVinfected versus HIV-uninfected adults in the United States: a cross-sectional study. J Am Heart Assoc. 2017;6(11):e007107.

47. Gilbert JM, Fitch KV, Grinspoon SK. HIV-related cardiovascular disease, statins, and the REPRIEVE Trial. Topics in antiviral medicine. 2015:23(4):146-9.

48. Mitka M. Exploring statins to decrease HIV-related heart disease risk. Jama. 2015:314(7):657-9.

49. Gaziano TA, Bitton A, Anand S, Abrahams-Gessel S, Murphy A. Growing epidemic of coronary heart disease in low- and middle-income countries. Curr Probl Cardiol. 2010;35(2):72-115.

50. Miranda JJ, Kinra S, Casas JP, Davey Smith G, Ebrahim S. Non-communicable diseases in low- and middle-income countries: context, determinants and health policy. Tropical Med Int Health. 2008;13(10):1225-34.

51. Schmid CH, Stark PC, Berlin JA, Landais P, Lau J. Meta-regression detected associations between heterogeneous treatment effects and study-level, but not patient-level, factors. J Clin Epidemiol. 2004;57(7):683-97.

52. Mills EJ, Jansen JP, Kanters S. Heterogeneity in meta-analysis of FDG-PET studies to diagnose lung cancer. Jama. 2015:313(4):419.

53. Thompson SG, Higgins JP. How should meta-regression analyses be undertaken and interpreted? Stat Med. 2002;21(11):1559-73. 